\title{
Structural Optimization of Variable Section Slender Manipulator Based on Sensitivity Analysis Method
}

\author{
Zhong Luo, Le Liang, Yanyan Chen, Fei Wang
}

School of Mechanical Engineering and Automation, Northeastern University, Shenyang, 110004, China

bliangle2007@163.com(corresponding author)

Keywords: Structure optimization; Slender manipulator; Finite element; Sensitivity analysis method

\begin{abstract}
A parameter optimization method based on sensitivity analysis is presented for the structural optimization of variable section slender manipulator. Structure mechanism of a polishing robot is introduced firstly, and its stiffness model is established. Then, a design sensitivity analysis method and a sequential liner programming (SLP) strategy are proposed. In the beginning of the optimization, the design sensitivity analysis method can be used to select the sensitive design variables which can make the optimized results more efficient and accurate. And then, it can be used to improve the convergence during the process of the optimization. The design sensitivities are calculated using the finite difference method. The search for the final optimal structure is performed using the SLP method. Simulation results show that the structure optimization method is effective to enhance the stiffness of the manipulator, no matter when the manipulator suffers constant force or variable force. This work lays a theoretical foundation for the structural optimization for such manipulators.
\end{abstract}

\section{Introduction}

Structure optimization is concerned with finding optimal shape of a structure by the iterative process based on structural response analysis and sensitivity calculation. Remarkable progress has been achieved in structure optimization during the past three decades [1-3]. For new attempts for structure optimization, various methods have been developed. Viljoen and Visser discussed the application of finite element and genetic methods to design optimization of thin-walled panels considering buckling effects [4]. Kim and Chang proposed fixed grid based shape optimization inspired from topology optimization techniques [5]. Perezzan and Hernandez carried out the design sensitivity analysis of the normal stress in a flexural system [6]. Li et al. improved the efficiency for the stress sensitivity calculation beneficially by introducing an integrated virtual load system [7]. Jarmai et al published the works on design optimization of welded orthogonally stiffened cylindrical shells in which constraint functions were expressed in explicit forms, and some new mathematical optimization methods were used [8]. Jalal compared the continuum-discrete ( C-D ) and discrete-discrete (D-D) approaches in detail which presenting shape sensitivity formulations for design-dependent loadings [9]. Tae-Kyoung eliminated the limitations of a shell formulation based on NURBS or T-splines and extended T-spline FEM to the analysis of shells using a mapping scheme [10]. In the problems of shape optimal design, Seonho used exact geometric models to enhance shape sensitivity of the isogeometric framework [11]. Nevertheless, more efforts are still required, in both analytical and application aspects, toward the design optimization of a structure.

In this paper, a sensitivity analysis-based optimization program has been developed to perform structure optimization of a 3-DOF inner-wall grinding robot of the solid-propellant rocket engines, and the structure optimization of the variable section slender manipulator of the robot is performed. Firstly, the main structure mechanism of the grinding robot is presented, and the stiffness model of the manipulator is established. Then, a sensitivity analysis method is proposed which has two function for the structure optimization. The first function is to select the sensitive design variables by comparing the ratios of the mechanical performance sensitivities and mass sensitivity of the manipulator. Meanwhile another equally important aspect is to improve the convergence in the process of the optimization. Finally, the optimizing objective can be achieved using the SLD method. 


\section{Structure mechanism and stiffness model of the manipulator}

As shown in Fig. 1, the position adjusting mechanism of the inner-wall grinding robot consists of the big arm, the forearm and the wrist whose sections are rectangular thin-walled structure [12]. The length of each of the three parts is $l_{1}, l_{2}, l_{3}$ and the practical setup is shown in Fig. 2.

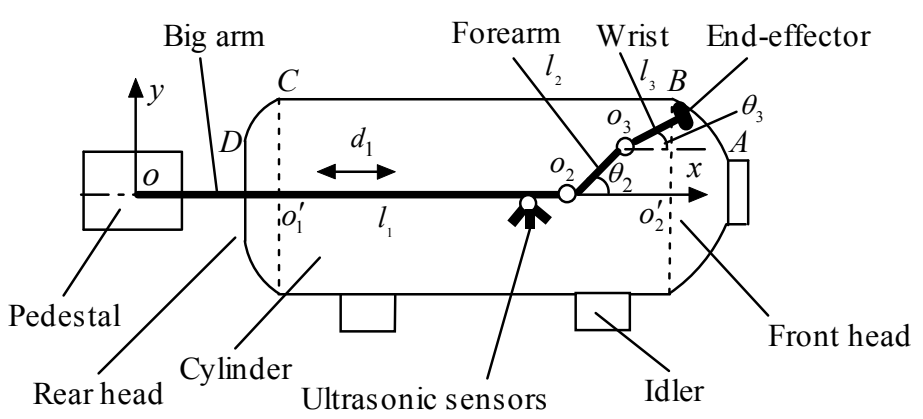

Fig.1 Schematic of the polishing robot

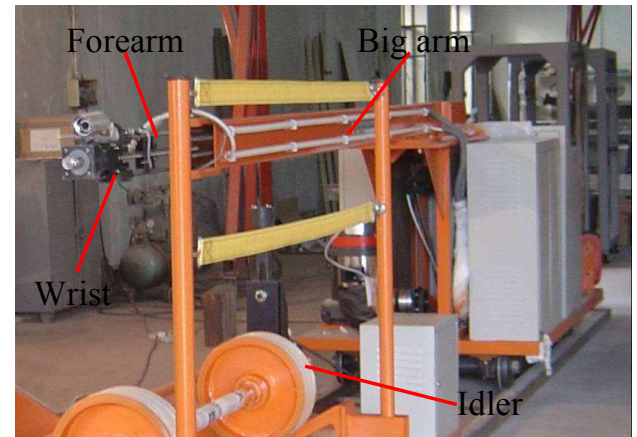

Fig.2 Experimental setup

The force diagram of the manipulator can be obtained after the force condition of the end-effector is applied to each joint. As shown in Fig.3, the elastic force is converted into one force and one equi-moment acting on each joint, similarly, the frictional force is converted into one force, one equi-moment and one equi-torque.

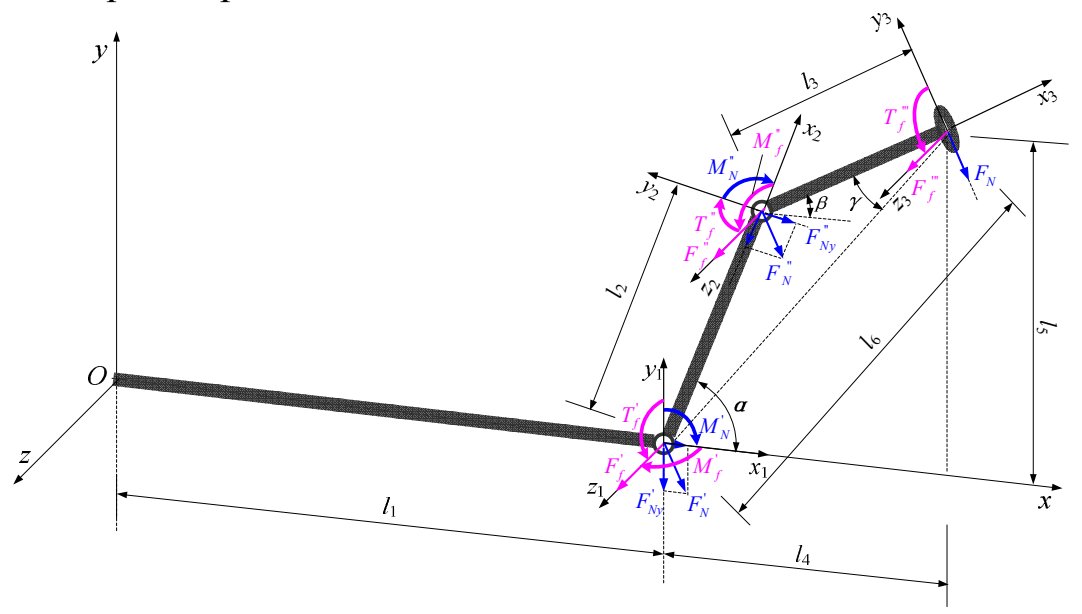

Fig.3 Force diagram of the manipulator

The bending defection of the manipulator under the force can be calculated as Eq (1).

$$
\boldsymbol{R}=\left[\begin{array}{l}
R_{x} \\
R_{y} \\
R_{z}
\end{array}\right]=\left[\begin{array}{ccc}
\frac{12 K_{3} F_{N}}{E} & \frac{12 K_{4} F_{N}}{E} & 0 \\
\frac{12 K_{1} F_{N}}{E} & \frac{12 K_{2} F_{N}}{E} & \frac{4 \cos \beta F_{N}}{K_{I, 3} E} \\
\frac{K_{5} F_{f}}{E} & \frac{K_{6} F_{f}}{E} & \frac{4 F_{f}}{K_{I, 3} E}
\end{array}\right]\left[\begin{array}{l}
\frac{l_{1}^{3}}{\delta_{1}^{4}} \\
\frac{l_{2}^{3}}{\delta_{2}^{4}} \\
\frac{l_{3}^{3}}{\delta_{3}^{4}}
\end{array}\right] .
$$

Where $\mathrm{E}$ is the elastic modulus of the material, $F_{N}$ is the elastic force, $F_{f}$ is the frictional force, $k_{I, i}$ is the scale factor of the rotational inertia which depends on the height-width ratio and the thicknesses of the manipulator, $K_{\mathrm{i}}$ is the dimensionless parameter which is related to the rotation angle and the length of the manipulator.

Based on the above calculation, the function relationship among the rotation angle of the forearm and the wrist $(\alpha, \beta)$, the wall thicknesses of the manipulator $\left(\delta_{1}, \delta_{2}, \delta_{3}\right)$, and the deformation $(\boldsymbol{R})$ can be obtained, so that the effect of wall thicknesses on stiffness of the manipulator is identified when the manipulator is in any working states. From the result of Eq. (1), we can see that a subtle change of the 
wall thickness can produce a relatively substantial influence on the deformation. Therefore the bending stiffness of the manipulator can be increased significantly through the optimal allocation of the wall thicknesses.

\section{Design sensitivity}

In this study, the first-order forward finite difference is used to calculate the design sensitivities of the objective function and constraint functions. The design variables of the analysis model are set to the wall thickness of the major parts of the manipulator $\left(x_{1} \sim x_{6}\right)$ and the length which is associated with the shape of cross $\operatorname{section}\left(x_{7} \sim x_{10}\right)$. The design sensitivities for objective function and constraint function can be written as

$$
S_{f, i}=\frac{\partial f}{\partial x_{i}}=\frac{f\left(x_{i}+\Delta x_{i}\right)-f\left(x_{i}\right)}{\Delta x_{i}}, \quad S_{\mathrm{g}, i}=\frac{\partial g_{j}}{\partial x_{i}}=\frac{g_{j}\left(x_{i}+\Delta x_{i}\right)-g_{j}\left(x_{i}\right)}{\Delta x_{i}} .
$$

Where $\Delta x_{i}$ is a small perturbation in the variable $x_{i}$.

Because the bigger the deformation of the manipulator, the smaller will be its stiffness, so the stiffness sensitivity can be instead by the displacement sensitivity. The sensitivities of some significant mechanical properties are performed for the design variables, which are listed in Table 1.

Table 1 Variable sensitivity results

\begin{tabular}{ccccccc}
\hline $\begin{array}{c}\text { Design } \\
\text { variable }\end{array}$ & $\begin{array}{c}\text { Initial } \\
\text { value[mm] }\end{array}$ & $\begin{array}{c}\text { Mass } \\
\text { sensitivity }\left(S_{\mathrm{m}}\right)\end{array}$ & $\begin{array}{c}\text { Bending } \\
\text { deflection } \\
\text { sensitivity }\left(S_{\mathrm{d}}\right)\end{array}$ & $\begin{array}{c}\text { Equivalent } \\
\text { stress } \\
\text { sensitivity }\left(S_{\sigma}\right)\end{array}$ & $\begin{array}{c}\text { Strain energy } \\
\text { sensitivity }\left(\mathrm{S}_{\mathrm{e}}\right)\end{array}$ & $\begin{array}{c}\text { Inherent } \\
\text { frequency } \\
\text { sensitivity }\left(S_{f}\right)\end{array}$ \\
\hline$x_{1}$ & 5 & $6.3234 \mathrm{e}-002$ & $6.9871 \mathrm{e}-002$ & 0.11418 & 0.16916 & 0.15739 \\
$x_{2}$ & 5 & $8.2416 \mathrm{e}-002$ & $2.5143 \mathrm{e}-002$ & $0.1716 \mathrm{e}-002$ & 0.11023 & $5.1693 \mathrm{e}-002$ \\
$x_{3}$ & 5 & $3.0099 \mathrm{e}-002$ & 0.22641 & 0.12743 & 0.15285 & $8.3761 \mathrm{e}-002$ \\
$x_{4}$ & 6 & $5.7318 \mathrm{e}-002$ & $2.3282 \mathrm{e}-002$ & $7.2927 \mathrm{e}-003$ & $3.5824 \mathrm{e}-002$ & $6.2573 \mathrm{e}-002$ \\
$x_{5}$ & 6 & 0.12734 & $0.2117 \mathrm{e}-002$ & $0.1253 \mathrm{e}-002$ & $4.4152 \mathrm{e}-002$ & $5.5148 \mathrm{e}-002$ \\
$x_{6}$ & 7 & 0.15828 & $1.8193 \mathrm{e}-002$ & $0.1496 \mathrm{e}-002$ & $1.3321 \mathrm{e}-002$ & $3.1549 \mathrm{e}-002$ \\
$x_{7}$ & 180 & $8.7849 \mathrm{e}-003$ & $3.0159 \mathrm{e}-002$ & $5.3381 \mathrm{e}-002$ & $4.8065 \mathrm{e}-002$ & $4.1746 \mathrm{e}-002$ \\
$x_{8}$ & 90 & 0.10628 & $3.6304 \mathrm{e}-002$ & 0.15539 & $8.1956 \mathrm{e}-002$ & $7.6385 \mathrm{e}-002$ \\
$x_{9}$ & 150 & 0.10923 & 0.23143 & 0.38164 & 0.26931 & 0.23372 \\
$x_{10}$ & 75 & $5.1605 \mathrm{e}-004$ & $0.1519 \mathrm{e}-002$ & $0.1618 \mathrm{e}-002$ & $0.18269 \mathrm{e}-002$ & $0.1516 \mathrm{e}-002$ \\
\hline
\end{tabular}

Though the bigger absolute value of the above sensitivities' the faster response of the corresponding variable is, the high sensitivity variable may reach the purpose of controlling the performance by increasing the mass of the structure, so it can not reflect the influence degree of the variables to the objective function or constraint functions accurately only with the value of the sensitivity. Based on the above consideration, the influence extent of each variable on the optimization objective can be assessed through comparing the ratios of the mechanical performance sensitivities and mass sensitivity of the manipulator which can be calculated as shown in Fig.4. Thus the major variables which have an important influence on the mechanical properties and a relatively insignificant affect on the mass can be found.

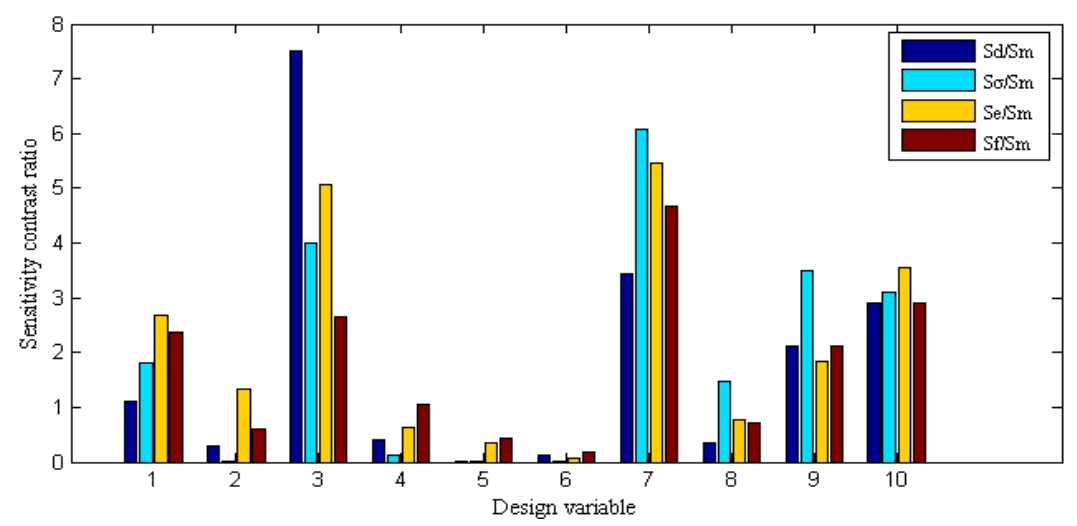

Fig.4 Contrast of sensitivities 


\section{Optimization model and solution algorithms}

In this study, sequential linear programming (SLP) is used to minimize the objective function with respect to the constraint equations. The objective function takes for the bending stiffness. When using the software to analysis, the function can be changed to the bending deflection $R$. The constraint conditions are set to four parts. (1) The mass of the manipulator must be controlled in certain scope. (2) The maximum equivalent stress meets the structure strength requirement. (3) The strain energy can not exceed the limit value. (4) In order to reduce vibration, the manipulator is request to have a high inherent frequency; meanwhile, it should ensure that the inherent frequency can not close to the working frequency to lest produce resonance.

$$
\left\{\begin{array}{l}
\left(m_{\mathrm{b}}-m_{\mathrm{a}}\right) / m_{\mathrm{b}} \geq \Delta_{\mathrm{m}} \\
\frac{\sigma_{s}}{\sigma_{\max }} \geq n \\
U \leq U_{\varepsilon} \\
f_{\mathrm{d}} \leq f \leq \eta f_{\mathrm{w}}
\end{array}\right.
$$

Where $m_{\mathrm{b}}$ and $m_{\mathrm{a}}$ are the mass of the manipulator before and after optimization, $\Delta_{\mathrm{m}}$ is the variation rate of the mass. $\sigma_{\mathrm{s}}$ and $\sigma_{\max }$ are yield strength and maximum equivalent stress respectively, and $n$ is the safety factor. $U$ is the strain energy, $U_{\varepsilon}$ is the limit value. $f, f_{\mathrm{d}}$ and $f_{\mathrm{w}}$ are defined as natural frequency, frequency lower limit and working frequency and $\eta$ is the limit coefficient of the frequency range.

Fig. 5 exhibits the evolutionary history of the bending deflection of the manipulator, which decreases from $1.3139 \mathrm{~mm}$ to $0.6699 \mathrm{~mm}$, i.e. by about $49.01 \%$. Through the process of optimization the optimal design scheme is listed in Table 2.

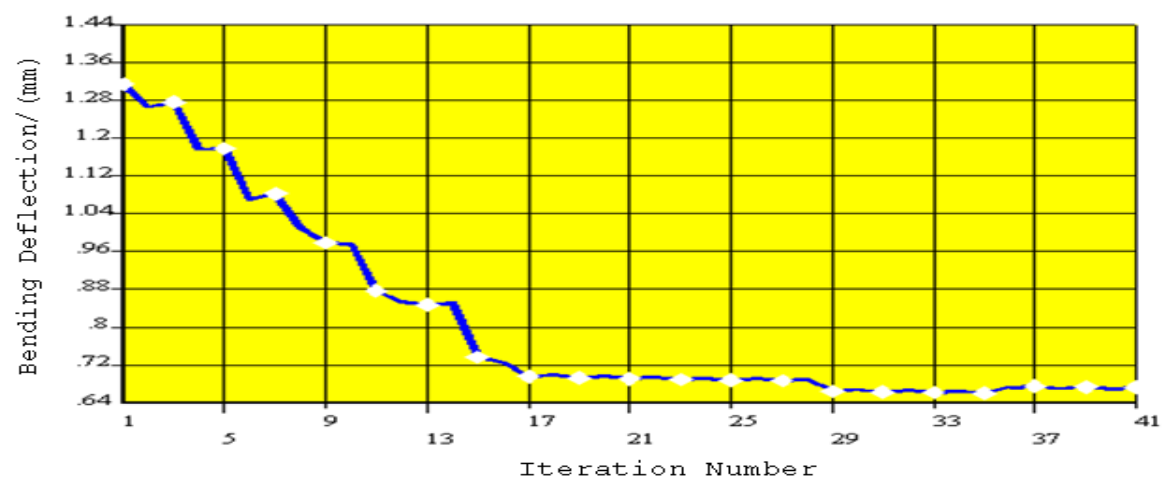

Fig. 5 Evolutionary history of the bending deflection in the design process

Table 2 Optimization design results

\begin{tabular}{cccccc}
\hline & Mass[kg] & $\begin{array}{c}\text { bending } \\
\text { deflection[mm] }\end{array}$ & $\begin{array}{c}\text { Equivalent } \\
\text { stress[Mpa] }\end{array}$ & $\begin{array}{c}\text { Strain } \\
\text { energy[mJ] }\end{array}$ & $\begin{array}{c}\text { Inherent } \\
\text { frequency[Hz] }\end{array}$ \\
\hline Initial value & 74.211 & 1.3139 & 20.007 & 44.281 & 130.47 \\
\hline Optimization result & 67.464 & 0.6699 & 15.398 & 42.744 & 120.631 \\
\hline
\end{tabular}

Comparing the optimization result listed in Table 2 can show that the mechanical properties of the manipulator are greatly improved; meanwhile, the mass is reduced to $67.464 \mathrm{~kg}$ from $74.211 \mathrm{~kg}$ in original, a decrease of $9.1 \%$ which realize the lightweight of the manipulator.

The above optimization results are obtained when the manipulator is under static force. But in the actual working process, the load is changing with time due to the heterogeneity of the processing material, the vibration caused by the electromotor, and so on. Through the mechanical testing of the inner-wall grinding robot in its actual working process, it can be detected that the manipulator is subjected to a load changing as sine wave with time. The response curves of the displacement of the manipulator under the above variable load are obtained after the finite element analysis of the new model. The comparison of the above response curves before and after optimization is shown in Fig.6. 


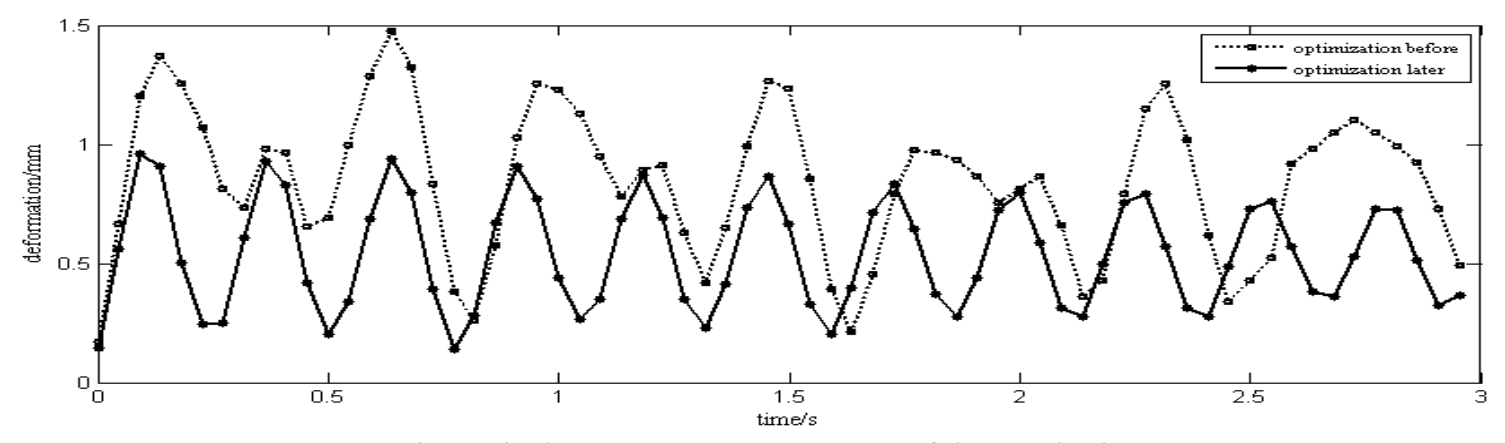

Fig.6 Displacement response curves of the manipulator

We can see from the Fig. 6 that the deflection of the manipulation is significantly declined, which is shown that the optimization method can be applied to the condition when the manipulator is subjected to a variable load.

\section{Conclusion}

In this paper, a structure optimization program has been developed for a 3-DOF inner-wall grinding robot of the solid-propellant rocket engines based on finite-element method and sensitivity analysis strategy. The sensitivity analysis method can be used not only to reduce the number of the design variables before optimizing for the purpose of increasing efficiency and accuracy, but also to improve the convergency during the process of optimization. The search for the optimal structure is performed using the SLP technique. This study is application-oriented and may be a useful example of structural design optimization for engineers.

\section{Acknowledgment}

This work was supported by National Science Foundation of China (51105064), Postdoctoral Science Foundation of China (20100481200), and Fundamental Research Funds for the Central Universities of China (N100403007).

\section{References}

[1] Vanderplaats GN, Numerial optimization techniques for engineering design: With application, McGraw-Hill, New York, 1984

[2] Haftka RT, Gurdal Z, Elements of structural optimization, Kluwer Academic Publishers, 1992.

[3] Pourazady $\mathrm{M}$ and $\mathrm{Fu} \mathrm{Z}$, An integrated approach to structural shape optimization, Computers and Structures, 60 (2) (1996), 279-289.

[4] Viljoen A and Visser A, Computationally efficient analysis and optimization of stiffened thin-walled panels in shear, Journal of Aircraft, 42 (3) (2005), 743-747.

[5] Kim. N. H and Chang. Y, Eulerian shape design sensitivity analysis and optimization with a fixed grid, Computer Methods in Applied Mechanics and Engineering, 194 (2005), 3291-3314.

[6] Perezzan JC and Hernandez S, Analytical expressions of sensitivities for shape variables in linear bending systems, Advances in Engineering Software, 34 (5) (2003), 271-278.

[7] Li Q, Steven GP, Xie YM, Evolutionary structural optimization for stress minimization problems by discrete thickness design, Computers and Structures, 78 (2000), 769-780.

[8] Jarmai K, Snyman J, Farkas, Minimum cost design of a welded orthogonally stiffened cylindrical shell, Computers and Structures, 84 (2006), 787-797.

[9] J. Akbari, N. H. Kim and M. T. Ahmadi, Shape sensitivity analysis with design-dependent loadings-equivalence between continuum and discrete derivatives, Structural and Multidisciplinary Optimization, 40 (2010), 353-364.

[10]Uhm T. K and Youn S. K, T-spline finite element method for the analysis of shell structures, International Journal for Numerical Methods in Engineering, 80 (2009), 507-536.

[11] Cho S and Ha S. H, Isogeometric shape design optimization: exact geometry andenhanced sensitivity, Structural and Multidisciplinary Optimization, 38 (1) (2009), 53-70.

[12]Zhong Luo, Shuxian Yang, Yilan Sun and Hongyi Liu, Optimized control for dynamical performance of the polishing robot in unstructured, Shock and Vibration, 18 (2011), 355-364. 